\title{
Bevacizumab treatment in the elderly patient with metastatic colorectal cancer
}

Clinical Interventions in Aging

5 January 2015

Number of times this article has been viewed

\section{Maria Di Bartolomeo \\ Claudia Maggi' \\ Francesca Ricchini' \\ Filippo Pietrantonio' \\ Roberto lacovelli' \\ Filippo de Braud' \\ Alessandro Inno 2}

'Medical Oncology, Fondazione IRCCS Istituto Nazionale dei Tumori, Milan, ${ }^{2}$ Department of Medical Oncology, Sacro Cuore-Don Calabria Hospital, Negrar, Italy
Correspondence: Maria Di Bartolomeo Medical Oncology, Department of Medical Oncology, Fondazione IRCCS Istituto Nazionale dei Tumori, Via Venezian I, 20133 Milano, Italy Fax +3902 23902149 Email maria.dibartolomeo@ istitutotumori.mi.it

\begin{abstract}
Metastatic colorectal cancer (mCRC), like many cancers, is primarily a disease of elderly people. Despite this prevalence, such patients are often excluded from randomized trials or represent a minority of enrolled patients. Moreover, the criteria for establishing benefit or side effects of treatment strategies in this population are uncertain and not well recognized. Bevacizumab improves the outcome of $\mathrm{mCRC}$ when used in combination with standard firstline and second-line chemotherapy and beyond the first disease progression when given with a chemotherapy backbone different from that used in the precedent line. The particular toxicity profile of this antiangiogenesis agent (in particular hypertension, thromboembolic events, hemorrhage, and renal failure) may discourage its use in elderly patients with comorbidities. Data from subgroup analyses of randomized trials and the results of recent cohort studies suggest a significant benefit from the addition of bevacizumab to standard chemotherapy for elderly patients comparable with that observed in younger patients, except for the increased risk for thromboembolic events. Age alone should not be a barrier to use of bevacizumab, and further research with a more complete geriatric assessment should investigate the role of bevacizumab in elderly patients with mCRC to avoid undertreatment of this patient population due to a historical conservative approach.
\end{abstract}

Keywords: bevacizumab, elderly, metastatic colorectal cancer, antivascular treatment, review

\section{Introduction}

Colorectal cancer (CRC) is the third leading cause of cancer-specific death in females and the fourth in males worldwide. ${ }^{1}$ The incidence and mortality of CRC increases with advancing age, and the global burden of the disease is expected to grow further over the next few decades due to the increase in life expectancy. ${ }^{2}$ In the USA, approximately $60 \%$ of new cases of CRC and $70 \%$ of CRC-related deaths occur in patients aged 65 years and older. ${ }^{3}$ About $50 \%$ of patients diagnosed with early-stage disease will ultimately develop metastatic CRC (mCRC) in spite of radical surgery and adjuvant therapies, whereas $25 \%$ of patients present with de novo $\mathrm{mCRC}$ at diagnosis. Unfortunately, when metastatic disease occurs, the prognosis remains poor, even if survival has improved, often exceeding 24 months, due to the introduction of targeted agents. ${ }^{4}$

Despite the high prevalence of the disease in the elderly, this patient population has been historically excluded or underrepresented in most clinical trials because of stringent inclusion criteria. Moreover, elderly patients enrolled in trials are highly selected, and generally fitter than the average patients observed in daily clinical practice. As a result, there is not sufficient evidence on the appropriate management of elderly patients with mCRC. The safety and efficacy of standard chemotherapy in older patients seem to be comparable with that reported for younger individuals, ${ }^{5}$ but 
limited data are available about the tolerance and effectiveness of biologic agents in the elderly population.

Bevacizumab (Avastin ${ }^{\circledR}$, Genentech, South San Francisco, CA, USA) is a recombinant, humanized monoclonal antibody that specifically targets vascular endothelial growth factor. ${ }^{6}$ Bevacizumab improves progression-free survival (PFS) and overall survival (OS) of mCRC patients when added to chemotherapy in the first-line and second-line setting and beyond the first disease progression when given with a chemotherapy backbone different from that used in the front line. ${ }^{7-9}$ Bevacizumab has a particular toxicity profile including: cardiovascular events, such as hypertensive crisis, chronic heart failure, myocardial infarction, venous thromboembolic events, and arterial thrombotic events; cerebrovascular events, such as transient ischemic attack, stroke, and central nervous system hemorrhage; gastrointestinal perforation; fistula formation; wound healing complications; and nephrotic syndrome. ${ }^{10,11}$ These adverse events may be of some concern for medical oncologists when choosing treatment for elderly patients with mCRC, and an accurate risk-benefit balance should be carried out before planning the therapeutic strategy on an individual basis. The aim of this paper is to present the available evidence on the efficacy and safety of bevacizumab in elderly patients with $\mathrm{mCRC}$, in order to help clinicians select patients who are potentially good candidates for receiving a bevacizumabbased therapy.

\section{Literature search methodology}

The currently available evidence on the effectiveness and tolerability of bevacizumab in older patients derives from retrospective analyses of subgroups enrolled in clinical trials without an upper age limit, pooled analyses of randomized trials, observational community-based studies, and prospective, randomized clinical trials specifically assessing the efficacy and safety of bevacizumab in elderly patients. For this paper, the PubMed database was searched for papers published before January 2014 using "bevacizumab" AND "colorectal cancer" AND "elderly" as search terms.

\section{Subgroup analyses of randomized clinical trials}

In the AVF2107g study, a landmark, randomized, placebocontrolled, Phase III clinical trial, the addition of bevacizumab to an irinotecan-based chemotherapy as first-line treatment for mCRC significantly improved OS (20.3 versus 15.6 months, hazard ratio [HR] 0.66), PFS (10.6 versus 6.2 months, HR 0.54$)$ and response rate (44.8\% versus $34.8 \%) .{ }^{7}$ Subgroup data analysis suggested a comparable survival benefit (HR 0.61 ) for patients aged $\geq 65$ years, who represented $32 \%$ of the entire population enrolled in the study. In this trial, bevacizumab did not exacerbate chemotherapy-related toxicities, but was associated with an increased incidence of grade three hypertension (11\% versus $2.3 \%$ ) and gastrointestinal perforation $(1.5 \%$ versus $0 \%)$. The incidence and grade of bevacizumab-related adverse events were not reported by age group, and concerns remained regarding the safety profile of bevacizumab in elderly patients. ${ }^{7}$

More information on the tolerability of bevacizumab in older patients was provided by the randomized, double-blind Phase II AVF2192g study evaluating the addition of bevacizumab to fluorouracil/leucovorin in patients with a poor prognosis and deemed unfit for first-line irinotecan-based chemotherapy. ${ }^{12}$ The design of this study was based on a retrospective, exploratory analysis of a small randomized Phase II trial (AVF0780) ${ }^{13}$ comparing 5-fluorouracil/folinic acid alone or combined with two different bevacizumab doses ( 5 or $10 \mathrm{mg} / \mathrm{kg}$ ), which suggested a particularly meaningful clinical benefit from anti-vascular endothelial growth factor therapy in a subset of patients with poor prognostic features, including age $\geq 65$ years, Eastern Coast Oncology Group performance status $\geq 1$, and albumin $\leq 3.5 \mathrm{~g} / \mathrm{dL}{ }^{13}$ As a result of the eligibility criteria used for the AVF2192g trial, $80 \%$ of patients were aged $\geq 65$ years, and the mean age was 70.7 and 71.3 years in the fluorouracil-leucovorin arm and fluorouracil-leucovorin plus bevacizumab arm, respectively. ${ }^{12}$ Patients receiving bevacizumab had a significantly longer median PFS (9.2 versus 5.5 months; HR $0.5 ; P=0.0002),{ }^{12}$ but the study did not meet the primary endpoint of demonstrating a 39\% reduction in risk of death in the bevacizumab arm. However, the median OS was 3.7 months longer for patients treated with bevacizumab and chemotherapy in comparison with those who received chemotherapy plus placebo (16.6 versus 12.2 months; HR $0.79 ; P=0.16) .{ }^{12}$ Despite its use in a population at high risk for serious adverse events, bevacizumab was well tolerated. Grade three hypertension was more frequent with bevacizumab (16\% versus $3 \%$ ) but was manageable with antihypertensive drugs, and did not lead to any discontinuation of treatment. There was no difference between the two groups in terms of incidence of bleeding and venous thromboembolic events, but an increased rate of arterial thrombotic events was observed in the bevacizumab arm when compared with the placebo arm (10\% versus $4.8 \%){ }^{12}$

Further information on use of bevacizumab in the elderly population derives from subgroup analyses of 
other randomized studies in patients not selected for age. In the Phase III BICC-C (Bolus Infusional, or Capecitabine with Camptostar-Celecoxib) study, $430 \mathrm{mCRC}$ patients were randomized to receive first-line FOLFIRI, modified irinotecan/fluorouracil/levoleucovorin (mIFL), or irinotecan plus capecitabine. ${ }^{14}$ After approval of bevacizumab, the study protocol was amended and an additional 117 patients, $24.8 \%$ of whom were elderly, were randomized to FOLFIRI or mIFL plus bevacizumab. The subgroup analysis did not show any significant difference in terms of efficacy and safety of bevacizumab plus chemotherapy for patients $\geq 65$ years compared with those $<65$ years. ${ }^{14}$

The randomized Phase III CAIRO-2 study (ClinicalTrials.gov identifier NCT00208546) investigated the addition of the anti-epidermal growth factor receptor monoclonal antibody cetuximab to bevacizumab plus capecitabine and oxaliplatin (CAPOX) in previously untreated mCRC patients. ${ }^{15}$ The study reported negative results, since the combination of the two monoclonal antibodies with chemotherapy determined a shorter PFS and a poorer quality of life. A subgroup analysis performed in the control arm showed that the effect of bevacizumab plus CAPOX was similar in terms of median PFS across ages (10.6 versus 9.6 versus 13.5 months for patients aged $<75,70-75$, and $>75$ years, respectively; $P=0.908$ ), but a trend toward a worse median OS was observed in elderly patients (20.3 versus 17.6 versus 13.1 months for patients aged $<75,70-75$, and $>75$ years, respectively; $P=0.063) .{ }^{16}$ The incidence of grade three and four adverse events did not differ significantly among the three age groups. However, older patients discontinued treatment for unacceptable toxicity more frequently than younger patients, and it is not clear whether bevacizumab was the main reason responsible for early discontinuation in elderly patients, since the evaluation of bevacizumab-specific toxicity among the three age groups was not an object of the analysis. ${ }^{16}$ The Australasian Gastrointestinal Trials Group MAX study was a Phase II/III trial comparing capecitabine versus capecitabine plus bevacizumab versus capecitabine and mitomycin plus bevacizumab as first-line therapy for mCRC patients. ${ }^{17}$ The addition of bevacizumab to chemotherapy resulted in an improved PFS in comparison with capecitabine alone ( 5.7 months for capecitabine, 8.5 months for capecitabine and bevacizumab, and 8.4 months for capecitabine, mitomycin and bevacizumab; capecitabine versus capecitabine plus bevacizumab, HR $0.63 ; P<0.001$ ). Patients aged $>75$ years, who comprised $21 \%$ of the overall population enrolled in the study, achieved a comparable benefit in terms of PFS when treated with bevacizumab
(HR 0.53 for capecitabine versus capecitabine plus bevacizumab; $P=0.001) .{ }^{18}$ The effect of bevacizumab was not different between patients $>75$ and $\leq 75$ years, as suggested by an interaction test between treatment and age groups for response rate, PFS, and OS. Interestingly, no significant differences in bevacizumab-related toxicity were reported by age. However, in elderly patients receiving bevacizumab and chemotherapy, an increased rate of hand-foot syndrome was observed, and this finding may reflect the longer exposure to treatment in the bevacizumab-containing arm. ${ }^{18}$

\section{Pooled analyses from prospective trials}

Subgroup data analyses for age are limited to the small number of elderly patients enrolled in each trial, and generally lack the power to detect any significant difference in outcome between younger and older patients. Pooling data from different studies increases the sample size for the analysis, producing more reliable results.

A pooled analysis of data from the AVF2107g and AVF2192g studies included 439 patients aged $\geq 65$ years (276 patients $\geq 70$ years), and showed that bevacizumab was associated with a statistically significant and clinically meaningful improvement in median PFS (9.2 versus 6.2 months; HR $0.52 ; P<0.0001)$ and OS (19.3 versus 14.3 months; HR $0.70 ; P=0.006)$ in this elderly population, with a similar benefit observed also for patients aged $\geq 70$ years. ${ }^{19}$ The incidence of bevacizumab-associated adverse events was comparable with that observed in the overall study populations in the two trials, and did not result in a higher rate of treatment discontinuation.

Cassidy et $\mathrm{al}^{20}$ pooled data from the AVF2107g and AVF2192g trials with data from two randomized Phase III studies of oxaliplatin-based chemotherapy with or without bevacizumab in the first-line and second-line setting, respectively, ie, the NO16966 trial and the E3200 trial. In this analysis, data from 1,142 patients aged $\geq 65$ years and 712 patients $\geq 70$ years were compared with those from 1,864 patients $<65$ years. This analysis confirmed a remarkable survival advantage for older patients from the addition of bevacizumab to standard chemotherapy in comparison with chemotherapy alone. Indeed, bevacizumab prolonged median PFS from 6.7, 6.9, and 6.4 months to 9.5 (HR 0.59; $P<0.0001$ ), 9.3 (HR 0.58; $P<0.0001$ ), and 9.2 months (HR 0.54; $P<0.0001$ ), and median OS from 16.5, 15, and 14.1 months to 19.9 (HR $0.77 ; P<0.0001$ ), 17.9 (HR 0.85; $P=0.015)$, and 17.4 months $(0.79 ; P=0.005)$ for patients aged $<65$, $\geq 65$, and $\geq 70$ years, respectively. ${ }^{20}$ As shown 
by the difference among HRs for progression and for death (Table 1), the effect of bevacizumab on median OS was less pronounced than its effect on median PFS. Regarding the safety profile of bevacizumab, the pooled analysis revealed that most of the bevacizumab-associated adverse events in the elderly population were not more frequent than those observed in younger patients. However, the incidence of arterial thrombotic events was higher in elderly patients receiving bevacizumab than in those treated with chemotherapy alone ( $5.7 \%$ versus $2.5 \%$ for patients $\geq 65$ years, $6.7 \%$ versus $3.2 \%$ for patients $\geq 70$ years). ${ }^{20}$

\section{Data from randomized prospective clinical trials in geriatric populations}

AVEX (AVastin in the Elderly with Xeloda) was the first trial evaluating the efficacy and safety of the addition of bevacizumab to chemotherapy specifically in an elderly population (Table 1). ${ }^{21}$ In this open-label Phase III study, 280 patients aged $\geq 70$ years with previously untreated $\mathrm{mCRC}$ and deemed not candidates for oxaliplatin-based or irinotecan-based chemotherapy were randomized to receive capecitabine alone or capecitabine with bevacizumab. Patients were excluded from the study if they had clinically significant cardiovascular disease or a history of thromboembolic events within the previous 6 months. The median age was 77 years in the control group and 76 years in the bevacizumab group, and about $50 \%$ of patients had an Eastern Coast Oncology Group performance status $\geq 1$. The response rate and disease control rate were significantly higher in the combination arm ( $19.3 \%$ versus $10 \%$, respectively, $P=0.042$; and $74.3 \%$ versus $57.9 \%, P=0.005) .{ }^{21}$ Moreover, the addition of bevacizumab to capecitabine led to a 4-month gain in median PFS (9.1 versus 5.1 months; HR 0.53; $P<0.001$ ), which was the primary endpoint of the study. The effect of bevacizumab on PFS was consistent across all age groups, with HRs of $0.52,0.60$, and 0.36 for patients aged 70-74, $75-79$, and $\geq 80$ years, respectively. OS was longer for the bevacizumab arm compared with the capecitabine alone arm (20.7 versus 16.8 months), although the difference was not statistically significant. ${ }^{21}$ However, as the authors pointed out, the study was not powered to detect differences in OS. Treatment-related toxicities of grade $\geq$ three occurred more frequently in the bevacizumab-containing arm than in the control group ( $40 \%$ and $22 \%$, respectively), and treatment discontinuation because of adverse events was more common for patients treated with bevacizumab and capecitabine compared with those receiving capecitabine alone (41\% versus $26 \%$ ). Furthermore, venous thromboembolic events and hand-foot syndrome were more frequent in the combination arm than in the capecitabine arm. However, the overall incidence of thromboembolic events was not different from that reported for nonelderly patients in previous trials with bevacizumab.

\section{Data from observational community-based trials}

Patients enrolled in clinical trials represent a highly selected population. They are often fit and without relevant comorbidities, and are different from the general population of elderly patients managed in daily clinical practice, who often have poorer performance status and additional risk factors. Observational studies provide an interesting opportunity to evaluate the efficacy and safety of bevacizumab in older patients outside of the clinical trials setting.

BRiTE (Bevacizumab Regimens: Investigation of Treatment Effects and Safety) was a prospective, observational study evaluating the efficacy and tolerability of bevacizumab and chemotherapy in a community-based setting. ${ }^{22}$ A subgroup analysis for age groups in this study showed that median PFS times were 9.6, 10, and 8.6 months for patients

Table I Main studies of the effectiveness of adding bevacizumab to standard chemotherapy in elderly patients with metastatic colorectal cancer

\begin{tabular}{|c|c|c|c|c|c|c|}
\hline Reference & $\mathbf{N} \%$ & Backbone CT & Line & $\mathbf{R R} \%$ & PFS (months) & OS (months) \\
\hline \multirow[t]{3}{*}{ Kabbinavar et $\mathrm{a}^{20}$} & $139 \mathrm{CT} / \mathrm{B}$ & $\mathrm{IFL} / \mathrm{FL}$ & I & 30.9 vs 25.5 & 9.2 vs 6.2 & 18.7 vs 12.6 \\
\hline & $137 \mathrm{CT}$ & & & NS & HR 0.5I & HR 0.69 \\
\hline & & & & & $P<0.0001$ & $P=0.015$ \\
\hline \multirow[t]{3}{*}{ Cassidy et $\mathrm{a}^{21}$} & $362 \mathrm{CT} / \mathrm{B}$ & IFL/FL/ & $1 / 2$ & NA & 9.2 vs 6.4 & I7.4 vs I4.I \\
\hline & $350 \mathrm{CT}$ & XELOXI & & & HR0.54 & HR 0.79 \\
\hline & & FOLFOX & & & $P<0.0001$ & $P=0.005$ \\
\hline \multirow[t]{3}{*}{ Cunningham et $\mathrm{al}^{22}$} & I $40 \mathrm{CT} / \mathrm{B}$ & Capecitabine & 1 & 19.3 vs 10.0 & 9.1 vs 5.1 & 20.7 vs 16.8 \\
\hline & $\mathrm{I} 40 \mathrm{CT}$ & & & $P=0.04$ & HR 0.53 & HR 0.79 \\
\hline & & & & & $P<0.001$ & $P=0.18$ \\
\hline
\end{tabular}

Abbreviations: $\mathrm{CT}$, chemotherapy; $\mathrm{B}$, bevacizumab; RR, response rate; PFS, progression-free survival; OS, overall survival; HR, hazard ratio; NA, not applicable; IFL, irinotecan/fluorouracil/levoleucovorin; FL, fluorouracil/levoleucovorin; XELOX, capecitabine/oxaliplatin; FOLFOX, 5-fluorouracil/leucovorin/oxaliplatin. 
aged $65-74,75-80$, and $>80$ years, comparable with the median PFS observed in younger patients ( 9.8 months) ${ }^{23}$ In contrast, the median OS decreased with age, from 26 months for patients $<65$ years to 16.2 months in patients $>80$ years. However, elderly patients receive all the active drugs for $\mathrm{mCRC}$ less frequently than younger patients, and it has been demonstrated that median OS is longer with exposure to all effective therapies. ${ }^{24}$ The safety profile of bevacizumab in the BRiTE study was similar for older and younger patients, with the exception of thromboembolic events, which increased significantly with age, occurring in $1.4 \%$ of patients aged $>65$ years, $4 \%$ of those aged $75-80$ years, and $4 \%$ of those aged $>80$ years. ${ }^{23}$ In the prospective, observational ARIES (Avastin Registry: Investigation of Effectiveness and Safety $)^{25}$ study, there was no difference between patients aged $<70$ years and $\geq 70$ years in terms of PFS both in first line (10.3 versus 9.9 months; HR 1.11) and second line (7.9 versus 7.9 months; HR 0.96). However, in this study, for patients receiving bevacizumab in first line, median OS was longer for younger patients (25.1 versus 19.6 months, HR 1.23$).{ }^{25}$

A cohort of 2,526 mCRC patients aged $\geq 65$ years treated from 2002 to 2007 with a fluoropyrimidine-based chemotherapy regimen as first line was identified by Meyerhardt et al within the Surveillance, Epidemiology and End Results (SEER) Medicare database. ${ }^{26}$ All the patients received a fluoropyrimidine-based chemotherapy regimen as first line. Addition of bevacizumab in 903 of these patients significantly prolonged median OS (19 versus 15.9 months; HR 0.87; $P=0.003$ ), but the benefit was limited to patients receiving irinotecan. Interestingly, patients treated with chemotherapy alone and those treated with chemotherapy and bevacizumab experienced a comparable rate of thromboembolic events within the first 6 months of treatment (17.1\% and 16.4\%), and the rate of cardiac events was higher in the chemotherapy group than in the bevacizumab group (14.5\% versus $11.5 \%)$. However, stroke occurred more frequently in patients receiving bevacizumab (4.9\% versus $2.5 \%$ ). ${ }^{26}$ The SEER database was also searched by Shankaran et al ${ }^{27}$ who analyzed data from patients aged $\geq 65$ years and diagnosed with mCRC between 2001 and 2007. Of 6,821 patients, 3,282 (48\%) were diagnosed in 2005-2007, and 19\% of them received first-line bevacizumab. In contrast with other reports, adverse event incidence rates in this analysis were comparable between patients in 2001-2003 receiving first-line chemotherapy (135 events per 100,000 person-days) and patients in 2005-2007 receiving first-line chemotherapy with bevacizumab (141 events per 100,000 person-days).

\section{Specific issues in the treatment of geriatric patients}

The optimal management of elderly patients with mCRC is still challenging. Assessment of geriatric patients considering only the "chronological age" may be inadequate to make a decision on the treatment strategy. Indeed, patients in the same age groups are often heterogeneous with respect to overall health status. Geriatric medicine has developed specific tools to detect some issues that can be missed by routine clinical and physical examination. A comprehensive geriatric assessment (CGA) includes social parameters, functional status and falls history, cognitive and psychological status, nutritional status, comorbidities, and polypharmacy. ${ }^{28}$ In elderly patients with cancer, several domains of CGA were demonstrated to correlate with oncological outcomes. Therefore, multiple organizations, including the International Society of Geriatric Oncology, ${ }^{29}$ have recommended that all older patients should undergo a CGA at the time of diagnosis and in the decision-making process. However, a CGA might be resource-consuming and time-consuming in daily practice, and rapid screening tests have been developed for a brief geriatric evaluation. ${ }^{30}$ Although CGA and rapid screening tests are prognostic tools, they have not been widely used in clinical trials as inclusion or stratification criteria. Also, in the AVEX trial, the only available Phase III study of bevacizumab in elderly patients, formal geriatric and comorbidity assessments were not performed. ${ }^{21}$ Another important issue when treating elderly patients with $\mathrm{mCRC}$, given the palliative intent of the therapy, is quality of life (QoL). Only limited data are available on the effect of bevacizumab on QoL in older patients. In the subgroup analysis of the CAIRO-2 study, QoL was not significantly different across age groups of patients treated with CAPOX plus bevacizumab; in this study, a 10-point increase in QoL was achieved in $17 \%, 15 \%$, and $33 \%$ of patients aged $<75$, $70-75$, and $>75$ years, respectively. ${ }^{16}$

\section{Conclusion}

Data from retrospective subgroup analyses and pooled analyses of randomized clinical trials, taken together with results from observational cohort studies, suggest a meaningful benefit from the addition of bevacizumab to standard chemotherapy for elderly patients with mCRC (Table 1$){ }^{31}$ The effectiveness of bevacizumab in older patients was comparable with that observed in younger patients. Recently, results from the AVEX trial confirmed a significant improvement of median PFS with the addition of bevacizumab to capecitabine. ${ }^{21}$ Bevacizumab is well tolerated, but its use 
may increase the risk of thromboembolic events, and the AVEX and the Australasian Gastrointestinal Trials Group MAX studies showed that it can also exacerbate hand-foot syndrome when given with capecitabine. ${ }^{18,21}$ Therefore, age alone should not be a barrier to use of bevacizumab, but treatment should be individualized, carefully selecting patients on the basis of medical history, with adequate assessment of clinically relevant comorbidities or risk factors, such as recent cardiovascular events, uncontrolled hypertension, or unstable angina. Furthermore, during treatment with bevacizumab, particular attention should be paid to monitoring and treating side effects in order to optimize the risk-benefit ratio. When patients are not properly selected, toxicity related to bevacizumab may be potentially more than expected. It should also be kept in mind that elderly patients treated outside of clinical trials are often frail, and that preservation of QoL is crucial in this setting. Further research should investigate the role of bevacizumab in elderly $\mathrm{mCRC}$ patients, with the incorporation of CGA, QoL assessment, and geriatric endpoints in clinical trials.

\section{Disclosure}

The authors report no conflicts of interest in this work.

\section{References}

1. Jemal A, Bray F, Center MM, et al. Global cancer statistics. CA Cancer J Clin. 2011;61(2):69-90.

2. Edwards BK, Howe HL, Ries LA, et al. Annual report to the nation on the status of cancer, 1973-1999, featuring implications of age and aging on U.S. cancer burden. Cancer. 2002;94(10):2766-2792.

3. Siegel R, Desantis C, Jemal A. Colorectal cancer statistics, 2014. CA Cancer J Clin. 2014;64(2):104-117.

4. Brenner H, Kloor M, Pox CP. Colorectal cancer. Lancet. 2014; 383(9927):1490-1502.

5. Folprecht G, Cunningham D, Ross P, et al. Efficacy of 5-fluorouracil-based chemotherapy in elderly patients with metastatic colorectal cancer: a pooled analysis of clinical trials. Ann Oncol. 2004;15(9):1330-1338.

6. Rosen LS. VEGF-targeted therapy: therapeutic potential and recent advances. Oncologist. 2005;10(6):382-391.

7. Hurwitz H, Fehrenbacher L, Novotny W, et al. Bevacizumab plus irinotecan, fluorouracil, and leucovorin for metastatic colorectal cancer. N Engl J Med. 2004;350(23):2335-2342.

8. Giantonio BJ, Catalano PJ, Meropol NJ, et al. Bevacizumab in combination with oxaliplatin, fluorouracil, and leucovorin (FOLFOX4) for previously treated metastatic colorectal cancer: results from the Eastern Cooperative Oncology Group Study E3200. J Clin Oncol. 2007;25(12):1539-1544.

9. Bennouna J, Sastre J, Arnold D, et al. Continuation of bevacizumab after first progression in metastatic colorectal cancer (ML18147): a randomised phase 3 trial. Lancet Oncol. 2013;14(1):29-37.

10. Tebbutt NC, Murphy F, Zannino D, et al. Risk of arterial thromboembolic events in patients with advanced colorectal cancer receiving bevacizumab. Ann Oncol. 2011;22(8):1834-1838.

11. Van Cutsem E, Rivera F, Berry S, et al. Safety and efficacy of firstline bevacizumab with FOLFOX, XELOX, FOLFIRI and fluoropyrimidines in metastatic colorectal cancer: the BEAT study. Ann Oncol. 2009;20(11):1842-1847.
12. Kabbinavar FF, Schulz J, McCleod M, et al. Addition of bevacizumab to bolus fluorouracil and leucovorin in first-line metastatic colorectal cancer: results of a randomized phase II trial. J Clin Oncol. 2005;23(16):3697-3705.

13. Kabbinavar F, Hurwitz HI, Fehrenbacher L, et al. Phase II, randomized trial comparing bevacizumab plus fluorouracil (FU)/leucovorin (LV) with $\mathrm{FU} / \mathrm{LV}$ alone in patients with metastatic colorectal cancer. $J$ Clin Oncol. 2003;21(1):60-65.

14. Jackson NA, Barrueco J, Soufi-Mahjoubi R, et al. Comparing safety and efficacy of first-line irinotecan/fluoropyrimidine combinations in elderly versus nonelderly patients with metastatic colorectal cancer: findings from the bolus, infusional, or capecitabine with camptostar-celecoxib study. Cancer. 2009;115(12):2617-2629.

15. Tol J, Koopman M, Cats A, et al. Chemotherapy, bevacizumab, and cetuximab in metastatic colorectal cancer. N Engl J Med. 2009;360(6): 563-572.

16. Venderbosch S, Doornebal J, Teerenstra S, et al. Outcome of first line systemic treatment in elderly compared to younger patients with metastatic colorectal cancer: a retrospective analysis of the CAIRO and CAIRO2 studies of the Dutch Colorectal Cancer Group (DCCG). Acta Oncol. 2012;51(7):831-839.

17. Tebbutt NC, Wilson K, Gebski VJ, et al. Capecitabine, bevacizumab, and mitomycin in first-line treatment of metastatic colorectal cancer: results of the Australasian Gastrointestinal Trials Group Randomized Phase III MAX Study. J Clin Oncol. 2010;28(19):3191-3198.

18. Price TJ, Zannino D, Wilson $\mathrm{K}$, et al. Bevacizumab is equally effective and no more toxic in elderly patients with advanced colorectal cancer: a subgroup analysis from the AGITG MAX trial: an international randomised controlled trial of capecitabine, bevacizumab and mitomycin C. Ann Oncol. 2012;23(6):1531-1536.

19. Kabbinavar FF, Hurwitz HI, Yi J, et al. Addition of bevacizumab to fluorouracil-based first-line treatment of metastatic colorectal cancer: pooled analysis of cohorts of older patients from two randomized clinical trials. J Clin Oncol. 2009;27(2):199-205.

20. Cassidy J, Saltz LB, Giantonio BJ, et al. Effect of bevacizumab in older patients with metastatic colorectal cancer: pooled analysis of four randomized studies. J Cancer Res Clin Oncol. 2010;136(5):737-743.

21. Cunningham D, Lang I, Marcuello E, et al. Bevacizumab plus capecitabine versus capecitabine alone in elderly patients with previously untreated metastatic colorectal cancer (AVEX): an open-label, randomised phase 3 trial. Lancet Oncol. 2013;14(11):1077-1085.

22. Kozloff M, Yood MU, Berlin J, et al. Clinical outcomes associated with bevacizumab-containing treatment of metastatic colorectal cancer: the BRiTE observational cohort study. Oncologist. 2009;14(9):862-870.

23. Kozloff MF, Berlin J, Flynn PJ, et al. Clinical outcomes in elderly patients with metastatic colorectal cancer receiving bevacizumab and chemotherapy: results from the BRiTE observational cohort study. Oncology. 2010;78(5/6):329-339.

24. Grothey A, Sargent D, Goldberg RM, et al. Survival of patients with advanced colorectal cancer improves with the availability of fluorouracil-leucovorin, irinotecan, and oxaliplatin in the course of treatment. J Clin Oncol. 2004;22(7):1209-1214.

25. Bendell JC, Bekaii-Saab TS, Cohn AL, et al. Treatment patterns and clinical outcomes in patients with metastatic colorectal cancer initially treated with FOLFOX-bevacizumab or FOLFIRI-bevacizumab: results from ARIES, a bevacizumab observational cohort study. Oncologist. 2012;17(12):1486-1495.

26. Meyerhardt JA, Li L, Sanoff HK, et al. Effectiveness of bevacizumab with first-line combination chemotherapy for Medicare patients with stage IV colorectal cancer. J Clin Oncol. 2012;30(6):608-615.

27. Shankaran V, Mummy D, Koepl L, et al. Adverse events associated with bevacizumab and chemotherapy in older patients with metastatic colorectal cancer. Clin Colorectal Cancer. 2013;12(3):204-213.

28. Solomon D, Sue Brown A, Brummel-Smith K, et al. National Institutes of Health Consensus Development Conference Statement: geriatric assessment methods for clinical decision-making. J Am Geriatr Soc. 2003;51(10):1490-1494. 
29. Extermann M, Aapro M, Bernabei R, et al. Use of comprehensive geriatric assessment in older cancer patients: recommendations from the task force on CGA of the International Society of Geriatric Oncology (SIOG). Crit Rev Oncol Hematol. 2005;55(3): 241-252.
30. Bononi A, Inno A, Borghi $\mathrm{M}$, et al. The prognostic value of rapid screening tests (RST) in geriatric assessment of patients with cancer over 70. J Clin Oncol. 2013;31 Suppl:Abstr 9554.

31. Sclafani F, Cunningham D. Bevacizumab in elderly patients with metastatic colorectal cancer. J Geriatr Oncol. 2014;5(1):78-88.
Clinical Interventions in Aging

\section{Publish your work in this journal}

Clinical Interventions in Aging is an international, peer-reviewed journal focusing on evidence-based reports on the value or lack thereof of treatments intended to prevent or delay the onset of maladaptive correlates of aging in human beings. This journal is indexed on PubMed Central, MedLine,

\section{Dovepress}

CAS, Scopus and the Elsevier Bibliographic databases. The manuscript management system is completely online and includes a very quick and fair peer-review system, which is all easy to use. Visit http://www.dovepress. $\mathrm{com} /$ testimonials.php to read real quotes from published authors. 